Claremont Colleges

Scholarship@ Claremont

All HMC Faculty Publications and Research

HMC Faculty Scholarship

$1-1-2003$

\title{
A Mathematical Model of Immune Response to Tumor Invasion
}

Lisette de Pillis

Harvey Mudd College

Ami Radunskaya

Pomona College

\section{Recommended Citation}

L.G. de Pillis and A.E. Radunskaya, "A Mathematical Model of Immune Response to Tumor Invasion", Computational Fluid and Solid Mechanics 2003, Proceedings of the Second M.I.T. Conference on Computational Fluid Dynamics and Solid Mechanics, pp. 1661-1668, June 2003.

This Article is brought to you for free and open access by the HMC Faculty Scholarship at Scholarship @ Claremont. It has been accepted for inclusion in All HMC Faculty Publications and Research by an authorized administrator of Scholarship @ Claremont. For more information, please contact scholarship@cuc.claremont.edu. 


\title{
A mathematical model of immune response to tumor invasion
}

\author{
L.G. de Pillis ${ }^{\mathrm{a}, *}$, A. Radunskaya ${ }^{\mathrm{b}, 1}$ \\ ${ }^{a}$ Harvey Mudd College, Department of Mathematics, Claremont, CA 91711, USA \\ ${ }^{b}$ Pomona College, Department of Mathematics, Claremont, CA 91711, USA
}

\begin{abstract}
Recent experimental studies by Diefenbach et al. [1] have brought to light new information about how the immune system of the mouse responds to the presence of a tumor. In the Diefenbach studies, tumor cells are modified to express higher levels of immune stimulating NKG2D ligands. Experimental results show that sufficiently high levels of ligand expression create a significant barrier to tumor establishment in the mouse. Additionally, ligand transduced tumor cells stimulate protective immunity to tumor rechallenge. Based on the results of the Diefenbach experiments, we have developed a mathematical model of tumor growth to address some of the questions that arise regarding the mechanisms involved in the immune response to a tumor challenge. The model focuses on the interaction of the NK and CD8 ${ }^{+} \mathrm{T}^{2}$ cells with various tumor cell lines using a system of differential equations. We propose new forms for the tumor-immune competition terms, and validate these forms through comparison with the experimental data of [1].
\end{abstract}

Keywords: Cancer; Tumor; Vaccine; Immunotherapy; Population models; Competition models; Mathematical modeling; Immune system; Ordinary differential equations

\section{Introduction}

A tumor's response to treatment depends on many factors, including the severity of the disease, the application of the treatment, and the strength of patient's own immune response. Mathematical modeling of this process is viewed as a potentially powerful tool in the development of improved treatment regimens. The mathematical modeling of tumor growth and treatment has been approached by a number of researchers using a variety of models over the past decades.

Therapeutic cancer vaccines have been studied recently in the medical community as a promising new adjuvant therapy. It is therefore important that we begin to develop mathematical models of tumor growth that include an immune system response, and ultimately a response to vaccine therapy.

To that end, we have developed a mathematical model of tumor-immune interactions to address some of the questions that arise regarding the mechanisms involved in the immune response to a tumor challenge. Our model is based upon and validated by recent experimental studies by

\footnotetext{
* Corresponding author. Tel.: +1 (909) 621-8975; Fax: +1 (909) 621-8366; E-mail: depillis@math.hmc.edu

${ }^{1}$ E-mail: aradunskaya@pomona.edu
}

Diefenbach et al. [1], in which mouse tumor cell lines are modified to express higher levels of immune stimulating NKG2D ligands. The experimental results show that sufficiently high levels of ligand expression create a significant barrier to tumor establishment in the mouse. Additionally, ligand transduced tumor cells stimulate protective immunity to tumor rechallenge. There is hope that these treatments with ligand-transduced tumor cells will eventually lead to the ability to effectively eliminate pre-established tumors, as well as naturally arising tumors.

Our mathematical model focuses in particular on the interaction of the $\mathrm{NK}$ and $\mathrm{CD}^{+} \mathrm{T}$ cells with various tumor cell lines using a system of differential equations. The model is used to explore the dynamics of tumor rejection, as well as to describe the development of protective immunity to subsequent tumor challenges.

The mathematical structure of the model is based upon earlier modeling work [2], in which tumor growth, an immune response, and chemotherapy treatment are represented by a system of four differential equations. Our representation also extends other lower-dimensional models, such as that described in [3] in which different cell populations are represented as interacting species. Other mathematical models that include an immune interaction with a tumor are described in [4-12]. 


\section{The model}

We focus on tissue near the tumor site, and we assume a homogeneous tumor. We will first present our biological assumptions, many of which are based upon the findings of [1]. We will then develop our model equations.

\subsection{Model equations}

The main conclusions of the experiments in [1] can be summarized as follows:

- NKG2D ligand expression (at sufficiently high levels)

- causes activation of $\mathrm{CD}^{+} \mathrm{T}$ cells and NK cells

- presents a significant barrier to tumor establishment

- Ligand transduced tumor cells can stimulate protective immunity to tumor rechallenge.

- Typical levels of NKG2D ligands naturally found on most tumor cell lines are suboptimal. It is hoped that tumor immunity can be boosted by engineering cells with higher ligand levels.

The specific biological assumptions we will take into account when developing our model equations are based both on accepted knowledge of immune system function as well as on conclusions stated in [1]. The assumptions include:

(1) The tumor cells grow logistically in the absence of an immune response. (This assumption is based on previous work, and is also suggested by graphs in [1] of tumor growth in mice without an immune response.)

(2) Both natural killer cells $(\mathrm{NK})$ and $\mathrm{CD}^{+} \mathrm{T}$ cells can kill tumor cells.

(3) Both NK cells and $\mathrm{CD}^{+}{ }^{+} \mathrm{T}$ cells are activated by tumor cells. Kill rates of both immune cell populations are increased when the tumor cells have been ligandtransduced. (This assumption is directly based on the findings of [1].)

(4) There is a background level of NK cells, even without any tumor being present.

(5) $\mathrm{CD}^{+} \mathrm{T}$ cells are not present in the absence of tumor cells.

(6) Both natural killer cells and $\mathrm{CD}^{+} \mathrm{T}$ cells eventually become inactivated after some number of interactions with tumor cells.

We will denote the three cell populations by:

- $T(t)$, tumor cell population at time $t$

- $N(t)$, natural killer cell population at time $t$

- $L(t), \mathrm{CD}^{+} \mathrm{T}$ cell population at time $t$

Using the above list of assumptions, we describe the system as three coupled differential equations:

$\frac{d T}{d t}=a T(1-b T)-F_{N}(T, N) T-F_{L}(T, L) T$

$\frac{d N}{d t}=e-f N+\frac{g T^{2}}{h+T^{2}} N-I_{N}(T, N)$ $\frac{d L}{d t}=-i L+\frac{j(\operatorname{dead} T)^{2}}{k+(\operatorname{dead} T)^{2}} L-I_{L}(T, L)$

The form of the equations was derived based on the principle of using the simplest form which would conform to the data. The two terms in the first equation, $F_{N}$ and $F_{L}$ represent the fraction of tumor cells killed in interactions with the two types of immune cells. Traditionally in the literature these competition terms are proportional to the competing populations, (see $[2,4,7]$ ). This form is generally justified by considering a cell-kinetic mechanism through which each immune cell has some fixed probability of encountering each tumor cell. In this model we will propose a different functional form for the interaction of tumor cells with the antigen-specific $\mathrm{CD}^{+}$cells.

Using the traditional form for the competition terms means letting $F_{N}=c N$ and $F_{L}=d L$ for some parameters $c$ and $d$ that need to be found through fitting with experimental data. However, when fitting for the parameters $c$ and $d$, we found that the linear product term produced growth curves for $T$ that were not a particularly good fit to the data provided in [1]. Instead, we found that we could produce curves that better fit the data by allowing

$F_{N}(T, N)=c N^{e_{N}} \quad$ and

$F_{L}(T, L)=d(L / T)^{e_{L}} /\left((L / T)^{e_{L}}+1\right)$

See Figs. 2 and 3.

The form of this term is supported by observations from [1]. In particular, both in vitro and in vivo experiments indicate that percent lysis appears to be a function of the ratio of $\mathrm{CD}^{+} \mathrm{T}$ cells to tumor cells, explaining the appearance of $(L / T)$. Furthermore, the data indicate that the percent of cells lysed never exceeds a maximum, resulting in the rational form given in Eq. (4).

The "recruitment" term in Eq. (2), $g T^{2} /\left(h+T^{2}\right)$, is of degree two, since, once again, this is the simplest form for the equation best fitting the data provided in [1]. For the NK cells, it is assumed that recruitment is a function of the total number of tumor cells in the system.

In Eq. (3), "dead $T$ " represents the number of tumor cells lysed by $\mathrm{CD}^{+} \mathrm{T}$ cells, i.e. $\operatorname{dead} T=F_{L} \cdot T$. The recruitment term, also a rational function of degree two, is a function of $\operatorname{dead} T$ alone, and does not depend directly on NK-cells or on the total amount of dead tumor cells in the system. This is because in [1] it is noted that it is ligand expression that results in the priming of tumor specific $\mathrm{T}$ cells, independent of the generation of cell debris by NK cells. Diefenbach et al. point out that although there are other studies which show that irradiated RMA cells can be effective in vaccinating mice, their own studies show that only mice vaccinated with irradiated ligand-transduced cells developed immunity. Those vaccinated with irradiated control-transduced tumor cells did not. Since it is understood that the $\mathrm{CD}^{+} \mathrm{T}$ cells are stimulated by the presence of tumor cells that have been recognized and processed 
by the immune system, our recruitment term is a function of the population of tumor cells that have already been attacked by $\mathrm{T}$ cells. In keeping with the Diefenbach observations, this recruitment term does not include the population of dead tumor cells generated by interactions with NK cells, or those which die naturally.

We also choose the "inactivation terms" to be

$I_{N}=p N T \quad I_{L}=q L T$

where $p$ and $q$ are parameters that have to be chosen to fit the experimental data. These simple forms for $I_{N}$ and $I_{L}$ are sufficient to allow for a good data fit, and represent the fact that immune cells are inactivated after some amount of interaction with tumor cells.

The model can be used to simulate the effect of enhancing ligand expression on tumor cells by allowing the relevant parameters to depend on the tumor-cell type. The relevant parameters in this model are $c$ and $d$, the effectiveness of the immune cells, along with $g$ and $j$, the recruitment parameters.

The parameters were estimated from the data in [1] by minimizing a least-squares distance using optimization software built into Matlab6. From [1] we were able to get data on the growth curves of different tumor cell types in the absence of an immune response, which allowed us to fit for parameters $a$ and $b$ (see Fig. 1). The competition parameters $c$ and $d$, as well as the exponents $e_{N}$ and $e_{L}$, were found by fitting to data that measured percent specific lysis as a function of $\mathrm{CD}^{+}$T-cell to tumor-cell or NK-cell to tumor-cell ratios. See Figs. 2 and 3. Data measuring percent of IFN- $\gamma$ producing immune cells as a function of ligand expression allowed us to fit for immune recruitment rates stimulated both by ligand-transduced and control-transduced tumor cells. Other parameters, such as the background source rate for NK-cells and death rates for immune cells, were taken from the literature, e.g. [3].

Figs. 4 and 5 show simulations which reflect the results in [1]. Ligand transduced cells stimulate the immune response enough to control tumor growth, while control-transduced tumor cells escape immune protection. In Fig. 5, the immune system is rechallenged after priming with ligand-transduced cells, showing the development of immunity.

The new forms for the competition terms we propose in Eq. (1) may suggest some insight about the mechanisms of the tumor-immune interactions. In contrast to NK cells, $\mathrm{CD}^{+} \mathrm{T}$ cells have to be primed by the tumor cells in order to be activated. Therefore, for the $\mathrm{CD}^{+} \mathrm{T}$-cell to tumorcell competition term, dead $T$, there were four different data sets to be fitted: priming with control-transduced cells and with ligand-transduced cells, then challenging with controltransduced and ligand-transduced cells. Experimental data indicated that the least effective combination was both to prime and to challenge with control-transduced cells, in which case there was at most around $10 \%$ lysis. In that case, the exponent $e_{L}$ was about 1 . The most effective combination was to prime and then challenge with ligandtransduced cells, resulting in a maximum of nearly $70 \%$ lysis. In this case, the exponent $e_{L}$ was closer to 0.56 . This may indicate that ligand expression allows for fewer interactions between $\mathrm{T}$ cells and tumor cells to be more effective.

\section{Summary}

Further simulations and analysis explore the possibilities of using ligand-transduced cells in vaccines, suggesting

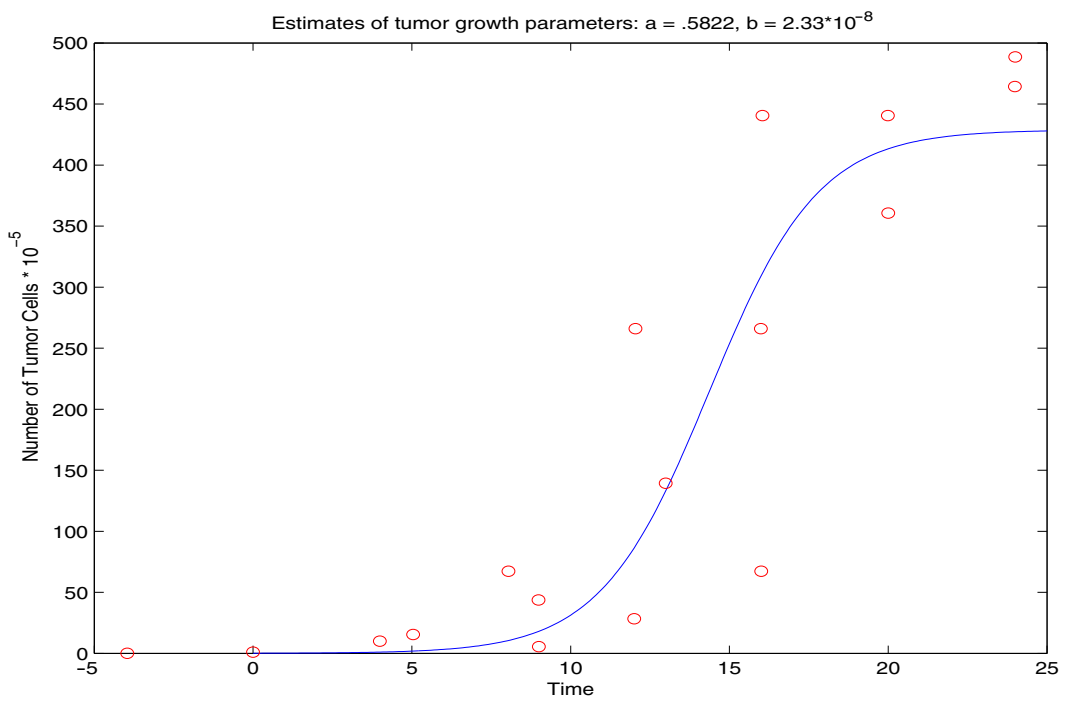

Fig. 1. Curve fit for parameters $a$ and $b$. 

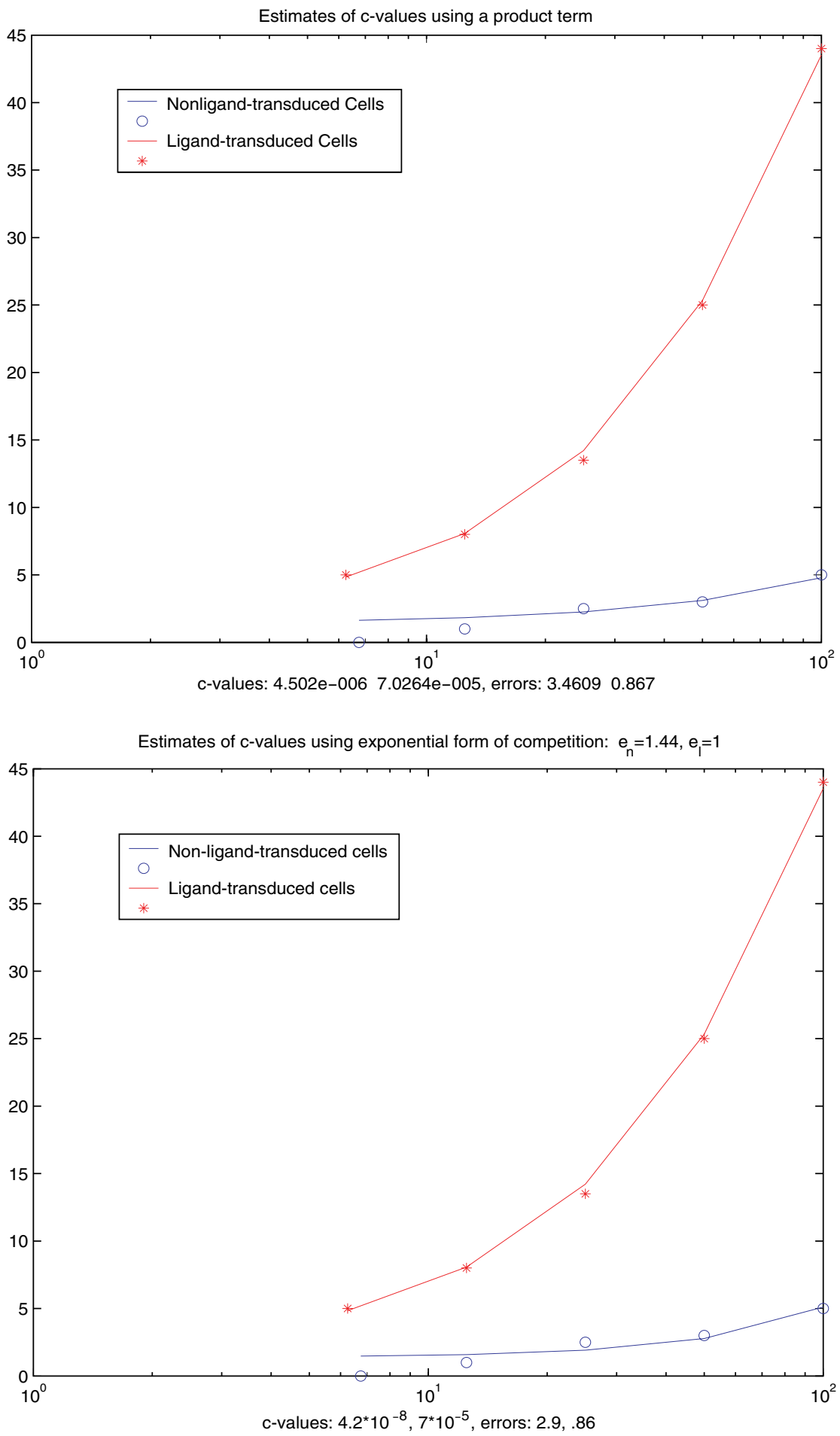

Fig. 2. Curve fit for the parameters $c$ both with and without ligands. Top: with a product competition term; bottom: with an exponential competition term. 

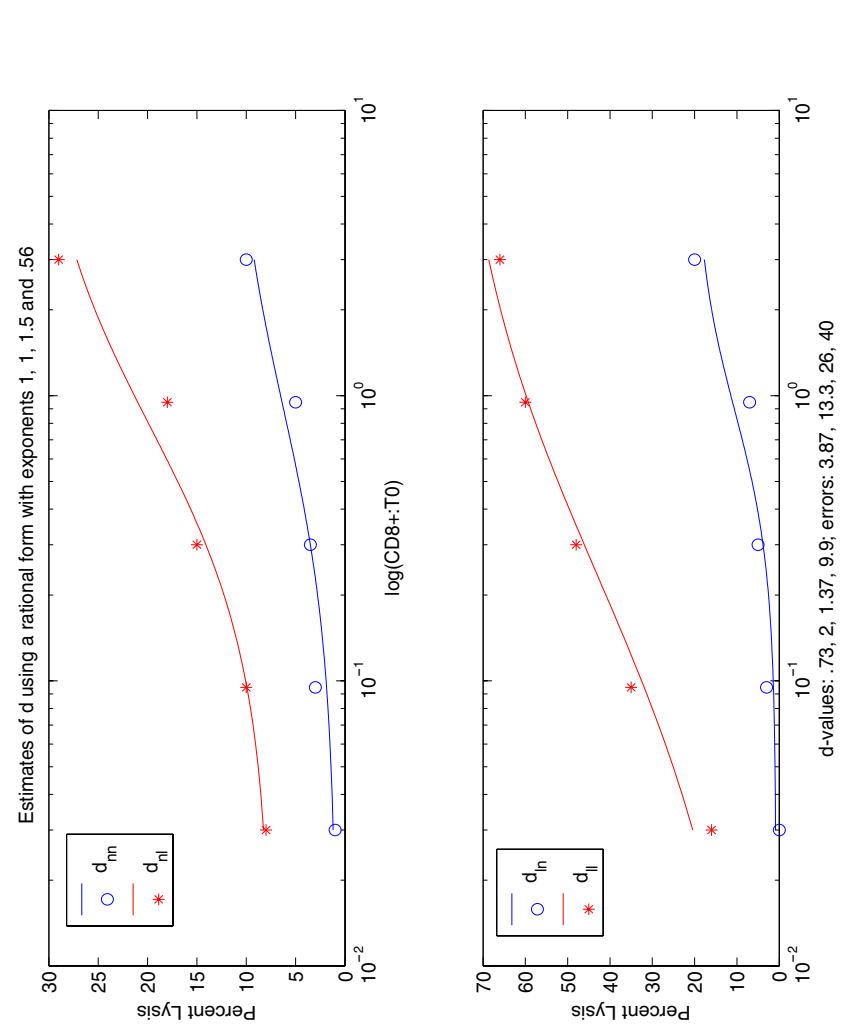

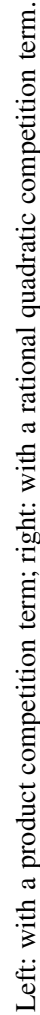
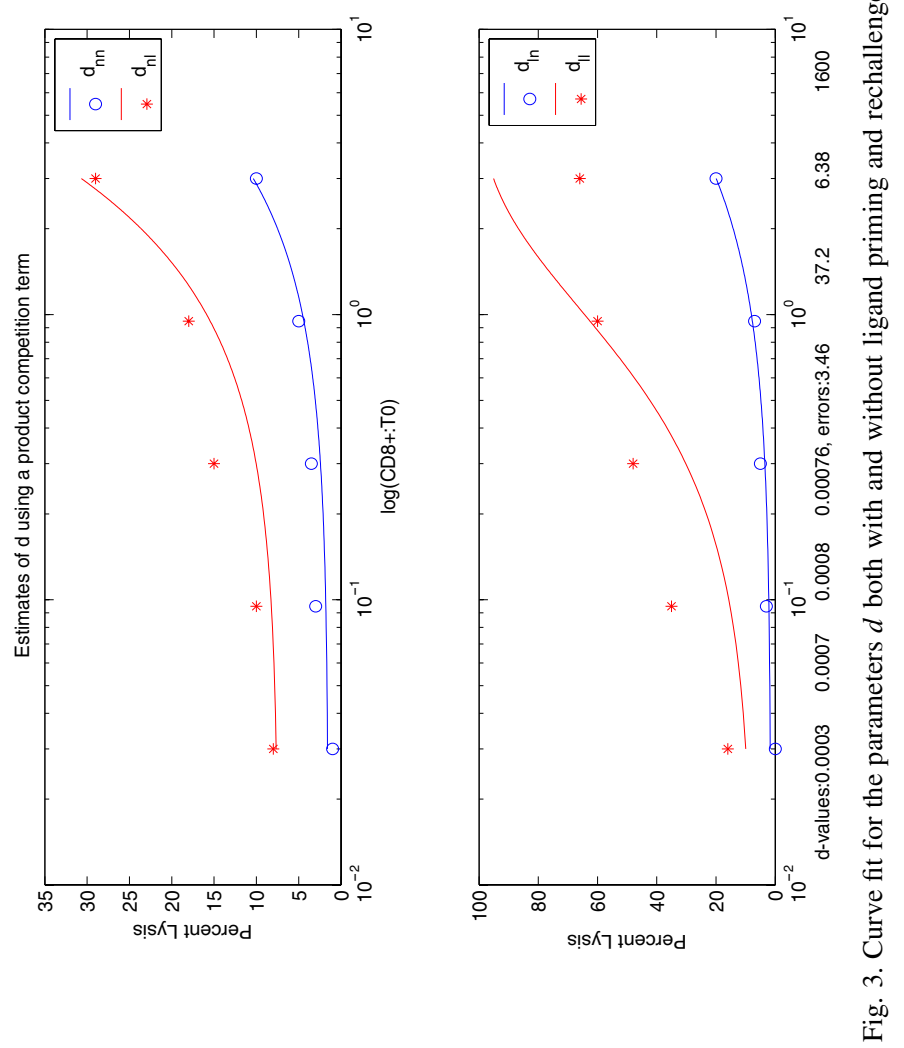

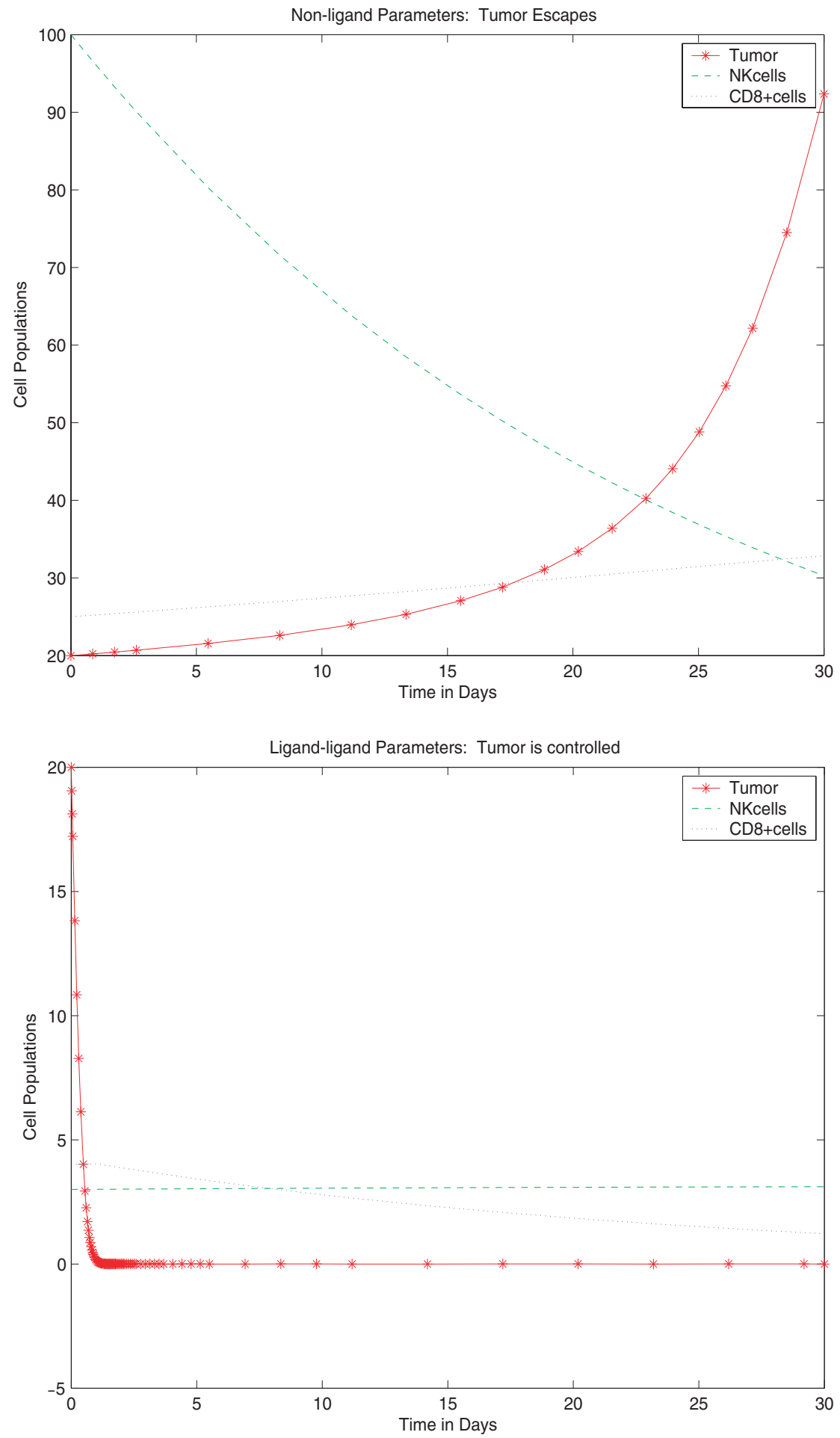

Fig. 4. Top: System evolution with tumor escape. Bottom: System evolution with tumor controlled. 

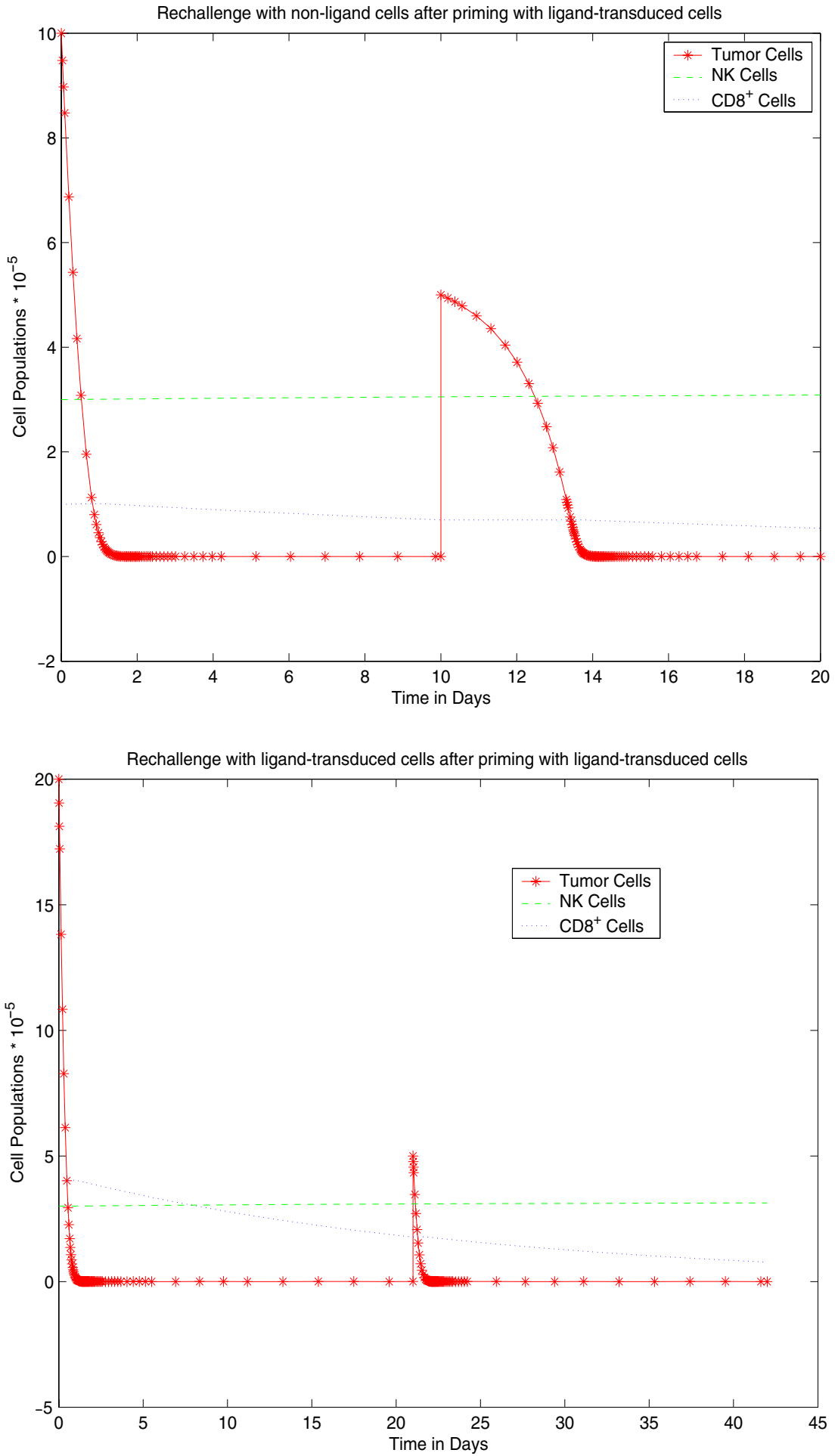

Fig. 5. Top: System evolution with non-ligand rechallenge. Bottom: System evolution with ligand-transduced rechallenge. 
optimal doses and timing of treatments. We note that this model presents tumor-immune interaction terms of a qualitatively different form than those commonly used. This form provides a good fit with experimental data resulting from priming and rechallenge by different combinations of tumor cell types. The mathematical model then suggests a rethinking of the mechanism by which $\mathrm{NK}$ cells and $\mathrm{CD}^{+}$ cells induce tumor cell lysis. These hypothetical mechanisms could be tested in the laboratory, allowing further refinements of the model and ideas for treatment.

\section{Acknowledgments}

We thank the members of the Mathematics of Medicine group at St. Vincent's Hospital in Los Angeles, especially Dr. Charles Wiseman and Dr. Tom Starbird. We also thank Dr. Jeff Rieker of Pomona Valley Hospital and Dr. Cymra Haskell for helpful discussions.

\section{References}

[1] Diefenbach A, Jensen E, Jamieson A, Raulet D. Rae1 and H60 ligands of the NKG2D receptor stimulate tumor immunity. Nature 2001;413:165-171.

[2] de Pillis L, Radunskaya A. A mathematical tumor model with immune resistance and drug therapy: an optimal control approach. J Theor Med 2001;3:79-100.
[3] Kuznetsov V, Makalkin I, Taylor M, Perelson A. Nonlinear dynamics of immunogenic tumors: Parameter estimation and global bifurcation analysis. Bull Math Biol 1994; 56(2):295-321.

[4] Kuznetsov V, Knott G. Modeling tumor regrowth and immunotherapy. Math Comp Model 2001;33:1275-1287.

[5] Bellomo N, Preziosi L. Modelling and mathematical problems related to tumor evolution and its interaction with the immune system. Math Comp Model 2000;32(3-4):413452.

[6] Firmani B, Guerri L, Preziosi L. Tumor/immune system competition with medically induced activation/deactivation. Math Models Meth Appl Sci 1999;4(9):491-512.

[7] Owen M, Sherratt J. Mathematical modelling macrophage dynamics in tumors. Math Models Meth Appl Sci 1999; 9(4):513-539.

[8] Kirschner D, Panetta J. Modeling immunotherapy of the tumor-immune interaction. J Math Biol 1998;37(3):235252.

[9] Michelson S, Leith J. Host response in tumor growth and progression. Invasion Metastasis 1996;16(4-5):235-246.

[10] Nani F, Oguztoreli M. Modeling and simulation of Rosenberg-type adoptive cellular immunotherapy. IMA J Math Appl Med Biol 1994;11(2):107-147.

[11] Adam JA. The dynamics of growth-factor-modified immune response to cancer growth: One-dimensional models. Math Comp Model 1993;17(3):83-106.

[12] Kuznetsov V, Makalkin I. Bifurcation-analysis of mathematical-model of interactions between cytotoxic lymphocytes and tumor-cells - effect of immunological amplification of tumor-growth and its connection with other phenomena of oncoimmunology. Biofizika 1992;37(6):1063-1070. 